\title{
ANALISIS DE RESOLUCIONES JUDICIALES SOBRE VIOLENCIA EN LA PAREJA EN LA POBLACIÓN MIGRANTE Y ESPAÑOLA EN ALMERÍA Y MURCIA (2005-2010)
}

\section{ANALYSIS OF JUDICIAL DECISIONS ON PARTNER VIOLENCE AMONG MIGRANT AND SPANISH POPULATION IN ALMERIA AND MURCIA (2005-2010)}

Pilar Rodríguez Martínez ${ }^{1}$ y Cristina Cuenca Piqueras ${ }^{2}$

Resumen: En este artículo llevamos a cabo una comparación de la incidencia de la violencia en la pareja entre poblaciones migrantes y autóctonas, del perfil socio-demográfico de agresores y víctimas, de los delitos por los que se condena, de las circunstancias especificas de los agresores, los lugares y testigos, que aparecen reflejados en las sentencias judiciales sobre violencia en la pareja entre la población migrante y española en el sureste español (Almería y Murcia) desde el año 2005 al 2010. La conclusión general del estudio es que las resoluciones judiciales reflejan y amplifican la violencia en la pareja que se produce entre las poblaciones migrantes en comparación con la que se produce entre la población autóctona.

\footnotetext{
Universidad de Almería.

2 Universidad de Almería.
} 
Palabras clave: Resoluciones judiciales, Violencia en la pareja, Almería, migrantes, españolas.

Abstract: In this article we carry out a comparison of the incidence of intimate partner violence among migrant and local populations, of the socio-demographic profile of offenders, of crimes, the specific circumstances of the aggressors, as well as places and witnesses, which are reflected in judicial decisions about intimate partner violence among migrant population in Southeast Spain (Almeria and Murcia) from 2005 to 2010. The overall conclusion of the study is that decisions reflect and amplify partner violence that occurs among migrant populations compared to that among native population.

Keywords: Judgments, partner violence, Almeria, migrants, Spanish.

\section{INTRODUCCIÓN}

En el presente trabajo realizamos una comparación del perfil socio-demográfico de agresores y víctimas, los delitos por los que se condena, las circunstancias específicas de los agresores, los lugares y testigos, que aparecen reflejados en las sentencias judiciales sobre violencia en la pareja entre la población migrante y española en el sureste español (Almería y Murcia) desde el año 2005 al $2010^{3}$.

Aquí nos referiremos a violencia en la pareja o expareja, y analizaremos tanto los supuestos en los que la víctima es mujer como cuando es varón, pudiendo ser esta violencia psíquica, física o sexual y, por tanto, englobando una multiplicidad de tipos delictivos entre los que se incluyen las amenazas, las coacciones, las lesiones, los malos

3 El trabajo de campo que está en la base de este artículo se desarrolló en el marco del proyecto de investigación «Violencia de género en poblaciones autóctonas y migrantes en el sureste español (Almería-Murcia). Un análisis comparativo", dirigido por Pilar Rodríguez y desarrollado en el Centro de Estudios para las Migraciones y las Relaciones Interculturales (Secretaria de Estado e Inmigración - Universidad de Almería) y en el que Cristina Cuenca participó como apoyo de investigación. Nos gustaría agradecer el trabajo exhaustivo llevado a cabo por los-as revisores-as de la revista Migraciones, que nos ha permitido mejorar, y en algunos casos, reformular, parte de nuestro análisis. 
tratos ocasionales y el maltrato habitual. Otros términos que han sido usados para conceptualizar el tipo de violencia a la que nos referimos son: violencia contra las mujeres, violencia de género, violencia doméstica o familiar, e incluso terrorismo doméstico ${ }^{4}$. Sin embargo, desde nuestro punto de vista, el concepto de violencia de género no es asumible para la investigación porque supone partir de que la posición desigual de la mujer es la única causa de la violencia. No explica por qué hay hombres que ejercen la violencia y otros que no, y por qué hay grupos de mujeres que sufren más esa violencia que otros (Larrauri, 2007:24; García, 2009:71; Rodríguez, 2010). En todo caso, la afirmación de que la violencia en la pareja se produce por múltiples motivos no implica que no se reconozca la influencia en este fenómeno de la desigual situación entre hombres y mujeres. Tal y como manifiesta García, «es perfectamente posible reconocer la pervivencia del patriarcado y la actualidad de la dominación masculina, así como entender que forman el caldo de cultivo en el que se da la violencia de género, sin tener que aceptar que nos dan su explicación, pues ésta es bastante más compleja todavía» (2009:72).

Pues bien, en este artículo analizamos las resoluciones judiciales que tienen que ver con la violencia en la pareja y expareja. En lo que se refiere al análisis socio-jurídico de resoluciones judiciales hay que destacar que pese a que se encuentran investigaciones de este tipo en otros países como, por ejemplo, en Canadá (Varma, 2002), en Estados Unidos de América del Norte (en adelante EEUU) (Kingsnorth, y MacIntosh, 2007), Australia (Shea y Bagshaw, 2008), Portugal (Mihai, 2010) y Reino Unido (Cederborg y Lamb, 2006) este tipo de investigación ha sido desarrollado en muy pocas ocasiones en

4 Como destaca Barrére, la expresión «violencia contra las mujeres» es un concepto hecho político por el movimiento feminista (2008:28-30). En cuanto al término "violencia familiar», el Consejo de Europa (1985) la define como: «Todo acto u omisión sobrevenido en el marco familiar por obras de uno de sus componentes que atente contra la vida, la integridad corporal o psíquica, o la libertad de otro componente de la misma familia, o que amenace gravemente el desarrollo de su personalidad». Respecto al denominado «terrorismo patriarcal», Johnson argumenta que en EEUU se producen dos formas de violencia sobre la mujer, la violencia en la pareja común, (que sería una violencia ocasional por parte de los maridos, de las esposas, o de ambos), y el terrorismo patriarcal (cuando las familias viven aterrorizadas por una violencia masculina sistemática que es instrumental, es decir, dirigida a mantener el control y la subordinación, ejerciéndose principalmente sobre las mujeres) (Johnson, 1995:285). 
nuestro país (Calvo, 1995:17-18). En España sólo hemos localizado dos análisis sociojurídicos de resoluciones judiciales sobre violencia en la pareja (Calvo 2001, Expósito et al., 2008). Ninguno se centra en estudiar dicho fenómeno entre las poblaciones migrantes.

De los estudios llevados a cabo en otros países se desprende que las denuncias y las resoluciones judiciales no tienen por qué corresponderse con la frecuencia o incluso las características de este fenómeno social. En esa línea, compartimos los planteamientos de Calvo (1995:9) y de Guemureman y Jorolinsky (2007:17), que parten de la idea de que los hechos legales - y entre ellos las decisiones judiciales- constituyen una construcción social que refleja e incide en la realidad social.

El estudio de la violencia en la pareja desde el análisis sociológico de sentencias supone plantearse diversos interrogantes. El primero es hasta qué punto las resoluciones judiciales se corresponden con la existencia real del fenómeno pues se denuncia solo un $27,4 \%$ de la violencia en la pareja que se produce (Macroencuesta, 2011), y además se produce el sobreseimiento de buena parte de los casos. Por otro lado, destacaría la cuestión de si son las resoluciones judiciales totalmente imparciales u objetivas ${ }^{5}$. Hay estudios que resaltan que existen «criterios o factores extrajurídicos» que influyen en las decisiones de los jueces (Manzanos 2004; Guemureman y Jorolinsky, 2007).

\section{VIOLENCIA EN LA PAREJA E INTERSECCIONALIDAD}

En el ámbito internacional, la denuncia pública de la violencia doméstica se inicia a partir de 1993, tras la «Declaración para la eliminación de la violencia contra la mujer»(ONU, 1993). No obstante, pasarán algunos años hasta que los organismos internacionales sean conscientes de que determinados factores - como la raza, el origen étnico, la clase, la orientación sexual, la discapacidad y la nacionalidad, entre otros- hacen que algunas mujeres se encuentren en una situación de mayor vulnerabilidad (Secretario General de Naciones Unidas, 2006:122).

5 Esta cuestión ha sido denunciada por CEAR-Euskadi (2009). En la misma línea, para nuestro objeto de estudio, Amnistía Internacional (2012) denuncia la mala praxis en el transcurso de los procesos judiciales. 
En los inicios, las instituciones de la Unión Europea adoptaron un «enfoque unitario» y centrado en el género, tanto para entender como para enfrentar la violencia que sufren las mujeres en las relaciones de pareja (Lombardo, 2009:9). En España también se impuso una "política monofocal». Sin embargo, en los estudios recientes realizados a nivel internacional y nacional se puede apreciar que hay determinadas mujeres que, debido a determinadas condiciones personales o sociales, tienen más posibilidades de sufrir violencia por parte de sus parejas o ex parejas. De modo que, como manifiestan Sokolof y Dupont, «la afirmación de que la violencia doméstica afecta por igual a toda persona, a través de raza, clase, nacionalidad, y religión no es sólo un gesto simbólico en la inclusión de diversas perspectivas sino también la prueba de una investigación descuidada en la construcción de teorías» (Sokoloff y Dupont, 2005:41). $\mathrm{Y}$ es que determinados colectivos sufren esta violencia con mayor frecuencia. En España, la última Macroencuesta (2011) refleja que las mujeres que tienen hijos, de entre 40 y 49 años, se encuentran desempleadas, o son extranjeras, se encuentran sobre representadas en las tasas de maltrato.

En el caso de las mujeres migrantes, como en el de las españolas, el estatus social se constituye a través de una complicada interacción entre clase, cultura, religión y otras instituciones y marcos de referencia. Esa idea ha sido sintetizada durante los últimos años en el concepto de interseccionalidad, que se refiere específicamente a la interacción entre el género, raza y otras categorías de diferencia en los distintos individuos, prácticas sociales, disposiciones institucionales, ideologías culturales y los resultados de estas interacciones en términos de poder (Mohanthy, 2008: 134; Cole, 2009). Estos planteamientos sobre la interseccionalidad están cada vez más presentes en el diseño de las políticas de género europeas (Secretario General de Naciones Unidas, 2006:122).

El enfoque interseccional asume que las causas de los malos tratos en la pareja son múltiples, y que en esa violencia pueden incidir diferentes factores simultáneamente que aumentarían el riesgo y la vulnerabilidad de las mujeres. Menjivar y Salcido (2002) destacan el desconocimiento de la lengua del país de llegada, su acceso al mundo laboral y el marco legal. Esas condiciones «se superponen a otros sistemas de opresión, como los de clase, raza y etnicidad, e incrementan la vulnerabilidad de estas mujeres a la violencia doméstica» (Menjivar y Salcido, 2002: 902). 
En esa línea, hay estudios que destacan la existencia de contextos socioculturales que pueden influir en la actuación de las víctimas migrantes. Se sugiere que algunas mujeres migrantes no acuden a los organismos en busca de ayuda porque las opciones que les facilitan pasan por dejar a su pareja. Este hecho, en algunas culturas, puede ser visto como una opción inaceptable para la mujer (Raj y Silverman, 2002: 384; Rubio, 2004:4; Malley-Morrison y Hines, 2007:953). Otras autoras destacan lo que denominan un «orden de silencio político», que impediría la denuncia de las situaciones de violencia en la pareja entre las poblaciones migrantes, y que consiste en una forma de presión de la comunidad para omitir información que pondría en entredicho la respetabilidad del grupo (West, 2005: 158).

Haciendo referencia a los factores económicos, multitud de estudios parten de la idea de que la precariedad aumenta las posibilidades de conflicto y violencia en las parejas (Dutton, Orloff y Hass, 2000; Heise y Garcia-Moreno, 2003; West, 2005; Hallyday y Lucas, 2010: 1142). En este sentido, Sokoloff y Dupont (2005:48) apuntan que, cuando los factores socioeconómicos son controlados, las diferencias raciales y étnicas en la tasa maltrato desaparecen en gran medida. Esta hipótesis de trabajo corroboraría los planteamientos feministas socialistas de autores como DeKeseredy y Schwartz (2002), cuando defienden un modelo teórico que explicaría el alto porcentaje de violencia sufrida por las mujeres en los barrios de vivienda subvencionada en EEUU. Atendiendo a los agresores, el estudio de Gondolf (2005) muestra que los hombres con identificación racial alta tenían más posibilidades de reincidir debido, precisamente, a que eran más pobres y con un nivel de estudios más bajo.

En lo que se refiere a España, las mujeres migrantes suelen incorporarse al mercado laboral en los peores empleos en tanto a condiciones laborales y salariales, y los nichos laborales en los que se insertan son principalmente el trabajo doméstico, la hostelería y la prostitución (Colectivo IOE, 1991; Oso, 1998; Parella, 2003, 2005; Morokvasick, 2007; Holgado, 2008). Esto supone que las migrantes tienen mayores dificultades para alcanzar una autonomía económica. La elección entre seguir soportando la violencia o vivir en la pobreza fue ya destaca por Dutton, Orloff y Hass en su estudio sobre mujeres latinas en EEUU (Dutton, Orloff y Hass, 2000: 250). Y, además, la pobreza se relaciona con mayores índices de consumo de alcohol entre la población de familias que pertenecen a las minorías 
étnicas en EEUU (West, 2005: 166; Moracco et al. 2005: 345; Gondolf, 2005:70; Kugel, 2009: 650).

Por otro lado, el estado civil y el número de hijos son factores que pueden intervenir en la frecuencia con que se experimenta violencia en la pareja. En este sentido, Heise y Garcia-Moreno señalan que la violencia doméstica es más común en las familias numerosas (2003:111). Halliday y Lucas destacan que las parejas de hecho presentan más problemas relacionados con la violencia en la pareja, precisamente porque la relación está menos institucionalizada y porque tienen menos recursos económicos (Halliday y Lucas, 2010:1143). También se ha observado que el embarazo constituye un momento crítico en el que aumenta la probabilidad de que las mujeres sufran violencia en la pareja (West, 2005:166).

La existencia o no de red social ha sido estudiada tratando de comparar la población migrante y autóctona. Los estudios señalan que las migrantes suelen estar más aisladas socialmente (Moracco et al., 2005: 339; Raj y Silverman, 2002:385; Menjivar y Salcido, 2002:904 Malley-Morrison y Hines, 2007:953). La falta de red social podría contribuir a aumentar el riesgo de sufrir la violencia y dificultaría, sobre todo, la búsqueda de apoyo en los servicios sociales, como destacan Raj y Silverman para el caso de las mujeres migrantes en EEUU que proceden del sur de Asia (2003: 435-437).

Respecto al acceso a los recursos que ofrece el Estado, los estudios insisten en que hay desigualdad para enfrentar una situación de violencia cuando se compara a las mujeres autóctonas y las migrantes (Dutton, Orloff y Hass, 2000:251). En el caso de España, Amnistía Internacional $(2005,2008)$, destaca que las migrantes se encuentran en una situación de desventaja respecto a mecanismos de denuncia y protección, a recursos especializados y a ayudas económicas.

La situación legal supone otro problema ya que el miedo a ser deportada es uno de los factores que impiden a las mujeres migrantes llevar a cabo la denuncia de la violencia (Dutton, Orloff y Hass, 2000: 252; Raj y Silverman, 2002:375-376; Menjivar y Salcido, 2002:908; Malley-Morrison y Hines, 2007:953). En el caso español hay que tener en cuenta que, en el período que aquí nos ocupa, el marco legal aplicable a las mujeres migrantes que han sufrido violencia en la pareja ha variado sustancialmente. Antes de la última reforma a la Ley Orgánica de Extranjería mediante la LO 2/2009 de 11 de diciembre, las migrantes en situación irregular podían solicitar una autorización de residencia (que no de trabajo), por ser víctimas de violencia 
de género, pero esta solicitud solamente se podía presentar en el momento en el que recayera sentencia de condena por delito (no por falta). Esto ocasionaba que las mujeres víctimas de violencia que estaban indocumentadas no iniciaban un proceso que podría tener como consecuencia su expulsión del territorio nacional, en el caso de que el agresor resultase absuelto. Además, el no poseer la autorización de trabajo les obligaba a trabajar sin contrato, por lo que tenían menos posibilidades de conseguir autonomía económica. La reforma realizada mediante LO 2/2009 de 11 de diciembre supone una mejora de condiciones, ya que se podía solicitar la autorización de residencia y también de trabajo por circunstancias excepcionales, una vez dictada sentencia de condena (sin distinguir entre delito o falta).

\section{OBJETIVOS DE LA INVESTIGACIÓN E HIPÓTESIS DE PARTIDA}

En nuestro trabajo partimos de la siguiente cuestión: ¿las resoluciones judiciales reflejan o amplifican la violencia en la pareja sufrida por las poblaciones migrantes respecto a las autóctonas? Como hipótesis general, y según se desprende de los estudios que hemos revisado, cabe pensar que las mujeres migrantes sufren más la violencia en la pareja que las españolas.

Además, los estudios revisados coinciden en resaltar que la violencia es ejercida por los varones hacia las mujeres, así que cabe esperar que en las resoluciones judiciales los agresores sean varones y las víctimas mujeres, tanto entre migrantes como entre españoles.

En segundo lugar, si bien West (2005) señala que las personas pertenecientes a las minorías tienen en común ser más jóvenes, menos educados y más pobres, -características asociadas a un mayor índice de violencia-, se han mencionado en el marco teórico estudios específicos sobre determinados colectivos (Dutton et al. 2000; Kugel et al. 2009; Moracco et al. 2005, en latinos; Gondolf, 2005, en afroamericanos) en los que se analizan las especificidades de cada minoría frente a la violencia. Por eso, cabe esperar que en las resoluciones judiciales haya diferencias entre los distintos continentes de procedencia de los y las migrantes.

En tercer lugar, Halliday y Lucas (2010) señalaban que las parejas de hecho, posiblemente por estar menos institucionalizadas 
y por tener menos recursos económicos, presentan más problemas de conflictividad, por lo que cabe suponer que en las resoluciones judiciales las parejas de hecho predominen sobre las casadas, tanto entre las poblaciones migrantes como entre la española.

En cuarto lugar, respecto al tipo de delitos ${ }^{6}$ por los que se les condena no contamos con ninguna referencia, por lo que vamos a suponer que las resoluciones judiciales no diferenciarán entre migrantes y españoles.

Según los estudios que hemos revisado, sólo Gondolf resaltaba que los hombres agresores con identificación racial alta tenían más probabilidades de reincidir que el resto, por lo que cabe esperar, en quinto lugar, que los migrantes agresores tengan más antecedentes penales que los españoles en nuestras resoluciones judiciales.

Respecto a los problemas psicológicos y relacionados con las drogas, hemos destacado que entre la población migrante puede haber mayor consumo y problemas psicológicos, por lo que cabe esperar, en sexto lugar, que encontremos una diferencia significativa entre los agresores migrantes y españoles en las resoluciones judiciales.

Por último, no disponemos de estudios que incidan en cuáles son las circunstancias en las que tiene lugar la violencia en la pareja comparando migrantes y autóctonos. Cabe esperar que, tanto entre migrantes como entre españoles, la violencia en la pareja se produzca sobre todo en el hogar familiar, por lo que los testigos que presenciarían la violencia en la pareja serían principalmente los hijos-as.

$\mathrm{El}$ análisis de sentencias que aquí presentamos consiste en revisar una por una las resoluciones judiciales, anotando quiénes son las personas que utilizan la violencia en sus relaciones de pareja, cuál es su sexo, los antecedentes penales de los agresores y las características del entorno en el que tiene lugar dicha violencia. Los casos que hemos incluido en nuestro análisis incluyen la totalidad de las

$6 \quad \mathrm{Al}$ respecto, se debe precisar que la violencia en la pareja aúna multitud de tipos delictivos. A pesar de que en otros estudios se utilicen los términos maltrato y malos tratos de forma aleatoria, desde un punto de vista legal hay importantes diferencias entre las dos formas de violencia. Por esto, cuando se habla de malos tratos ocasionales, nos referimos al tipo regulado por el artículo 153 del Código Penal, que consiste en menoscabos psíquicos o físicos sin que se haya causado lesión cuando la víctima es o ha sido su pareja. De otro lado, el maltrato habitual (tipificado por el artículo 173.2 del Código Penal), condenaría las mismas conductas pero cuando se producen con habitualidad (tal y como es definida por las SSTS de 24 de junio, de 7 de julio y de 7 de septiembre de 2000, entre otras). 
sentencias disponibles sobre violencia en la pareja y ex pareja en la provincia de Almería y en la región de Murcia desde el año 2005 al 2010, a partir de la base de datos Westlaw de Aranzadi ${ }^{7}$.

Partiendo de una rigurosa revisión de los textos, hemos llevado a cabo un análisis cuantitativo de 390 resoluciones judiciales emitidas en el ámbito territorial objeto de estudio. Esta base de datos es la más completa de las disponibles en la actualidad, pero una de sus principales limitaciones es que las sentencias que contiene no suelen citar la procedencia de agresores y victimas. Ha sido necesario completar el lugar de nacimiento de agresor y víctima, lo que hemos hecho gracias a la colaboración de la Administración de Justicia. Así que hemos dedicado un enorme esfuerzo a reconstruir los datos sobre el lugar de nacimiento ${ }^{8}$, así como a codificar las sentencias. Elegimos conscientemente iniciar nuestra búsqueda en el año 2005, teniendo en cuenta la fecha de publicación de LO 1/2004, de 28 de diciembre de medidas de protección integral contra la violencia de género.

\section{RESULTADOS DE LA INVESTIGACIÓN}

En nuestra investigación realizamos una comparación de nuestros datos con los datos disponibles sobre el volumen de la población migrante y los porcentajes de violencia en la pareja sufridos por mujeres que se desprenden de la Macroencuesta de Violencia de Género del año 2011.

7 La base de datos Westlaw de Aranzadi es la que recoge el mayor número de sentencias dentro del ámbito territorial señalado. Aranzadi incorpora a la base todas las resoluciones dictadas por órganos colegiados. Sin embargo, no es así en el caso de las sentencias dictadas por órganos unipersonales, incluyéndose aquellas que son incorporadas por el Centro de Documentación Judicial a la base de datos del Consejo General del Poder Judicial. Se incluyen las resoluciones que se consideran interesantes y son colgadas en su página Web como «Sentencias de actualidad»y aquellas que son solicitadas expresamente por algún usuario del sistema.

8 Hay que aclarar que en nuestra reconstrucción de la nacionalidad y el lugar de nacimiento de agresores-as y victimas no siempre hemos conseguido saber esos dos datos para cada una de las sentencias. A veces nos han facilitado ambos datos, a veces el lugar de origen aparece en el texto de la sentencia y, en otros casos, ha sido imposible aportar este dato. 
En lo que se refiere a las poblaciones migrantes y autóctonas de Almería y Murcia, hay que destacar que ambas provincias destacan por su gran dinámica migratoria en los últimos años. En concreto, y según el Instituto Nacional de Estadística, la Provincia de Almería y la Región de Murcia en 2010 tenían una población de 2.157.539 personas, de las que un 18,72\% habían nacido en el extranjero. Entre la población migrante, la mayoría procede de Europa $(38,82 \%)$, seguida por el continente africano $(31,04 \%)$, América $(28,4 \%)$, Asía $(1,69 \%)$ y Oceanía $(0,03 \%)$.

\subsection{Nacionalidad de origen, sexo y estado civil de agresores y víctimas}

Aunque la población migrante de Almería y Murcia supone un $18,72 \%$ del total de la población, la población migrante envuelta en problemas de violencia en la pareja supone un $28,84 \%$ respecto al total de población que aparece en las sentencias, cálculo que resulta de los datos que aparecen en la tabla 1. Los casi diez puntos de diferencia señalan que, por la razón que sea, las resoluciones judiciales se dictan con más frecuencia sobre personas migrantes que sobre españolas.

Así que las poblaciones migrantes sufren más la violencia en la pareja que las autóctonas, situación que reflejarían las resoluciones judiciales. Pero además, podemos decir que amplifican esa diferencia. Ese sobredimensionamiento se observa también si se comparan nuestros datos con los obtenidos en la Macroencuesta de Violencia de Género de 2011. Así, aunque no es fácil comparar estos datos ya que, como es sabido, dicha encuesta está basada en una muestra exclusivamente de mujeres (y para todo el territorio español), las mujeres de origen extranjero que aparecen en las resoluciones judiciales estudiadas aquí suponen un $35,21 \%$ de todas las víctimas de las que se conoce el lugar de nacimiento. Si tenemos en cuenta que las mujeres extranjeras que sufrieron violencia en la pareja según la Macroencuesta del 2011 suponían un 14,5\% del total de las mujeres que sufrían violencia en la pareja, podemos concluir -de nuevo-que las resoluciones judiciales contribuyen a generar una imagen que sobrerepresenta la violencia en la pareja de las migrantes frente a las españolas. Así pues, nuestra hipótesis general queda corroborada, pues las diferencias entre la población migrante y española son mucho mayores de lo que cabría esperar. 


\section{TABLA 1}

REGIONES DE ORIGEN DE AGRESORES Y VÍCTIMAS EN LAS SENTENCIAS RELACIONADAS CON LA VIOLENCIA FAMILIAR EN LA PROVINCIA DE ALMERÍA Y LA REGIÓN DE MURCIA (ENERO 2005-JULIO 2010) (390 CASOS).

\begin{tabular}{|c|c|c|c|c|c|c|}
\hline $\begin{array}{l}\text { PAÍS DE } \\
\text { ORIGEN }\end{array}$ & \begin{tabular}{|l|} 
HOMBRES \\
AGRESORES
\end{tabular} & \begin{tabular}{|l|} 
HOMBRES \\
VICTIMAS
\end{tabular} & \begin{tabular}{|l} 
RIÑA EN \\
PAREJA \\
(HOMBRE)
\end{tabular} & \begin{tabular}{|l|} 
MUJERES \\
VICTIMAS
\end{tabular} & \begin{tabular}{|l|} 
MUJERES \\
AGRESORAS
\end{tabular} & $\begin{array}{l}\text { RIÑA EN } \\
\text { PAREJA } \\
\text { (MUJER) }\end{array}$ \\
\hline Unión Europea & 16 & 1 & 1 & 9 & 1 & 1 \\
\hline Magreb & 19 & 1 & & 24 & 1 & \\
\hline América Latina & 58 & 1 & 1 & 56 & 1 & 1 \\
\hline $\begin{array}{l}\text { África } \\
\text { Subsahariana }\end{array}$ & 9 & & 1 & 3 & & 1 \\
\hline $\begin{array}{l}\text { Europa del } \\
\text { Este }\end{array}$ & 4 & & 1 & 2 & & \\
\hline España & 194 & 11 & 4 & 184 & 11 & 2 \\
\hline $\begin{array}{l}\text { Extranjero/a } \\
\text { no especifica } \\
\text { nacionalidad }\end{array}$ & 5 & & 1 & 6 & & \\
\hline $\begin{array}{l}\text { Total } \\
\text { nacionalidades } \\
\text { concretadas }\end{array}$ & 305 & 14 & 9 & 284 & 14 & 5 \\
\hline $\begin{array}{l}\text { Nacionalidad } \\
\text { no asignada }\end{array}$ & 58 & 2 & 2 & 84 & 2 & 1 \\
\hline Total & 363 & 16 & 11 & 368 & 16 & 6 \\
\hline
\end{tabular}

Fuente: Elaboración propia.

Nuestra primera hipótesis señalaba que la violencia es ejercida por los varones hacia las mujeres. Si observamos nuestros datos, la mayoría de los casos señalan que la víctima de la violencia es mujer y el agresor es varón, tanto entre españoles/as como entre extranjeros/as. Pero no siempre, como se puede observar en la tabla 1. En este sentido, las resoluciones judiciales avisan de la existencia de un tipo de violencia en la pareja -de mujeres hacia sus parejas varones- que, aunque minoritaria, habría que tenerla en cuenta. Dicha violencia puede ser ejercida tanto por migrantes como por españolas.

Nuestra segunda hipótesis se corrobora con los datos, ya que sí existen diferencias notables entrelos distintoslugares de procedencia 
de los y las migrantes. De hecho, entre la población migrante implicada destaca la procedente de América Latina (52,44\%), Magreb (20\%) y Europa (16\%). Sin embargo, entre las poblaciones migrantes que residen en este territorio, la mayor parte proceden de Europa, seguidos de África y América. De modo que, las resoluciones judiciales presentan una distribución de la violencia en la pareja que no tiene que ver con la cantidad de personas que habitan en este territorio y que provienen de ese continente. La razón no puede ser establecida porque no tenemos manera de conocer qué porcentaje de personas -o incluso sólo de mujeres- de cada procedencia que afirma haber sufrido violencia, ya que en la Macroencuesta no se especifica.

Sin embargo, en la tabla 1 hay otro dato a considerar: el hecho de que hay más mujeres víctimas que varones agresores del Magreb, mientras que entre los procedentes de Europa hay más agresores hombres que mujeres víctimas. Este dato resulta contradictorio si pensamos que en nuestro territorio hay más hombres que mujeres que proceden del Magreb y, en el caso de los que proceden de Europa, los hombres sólo sobrepasan ligeramente a las mujeres, como se puede observar en el Anexo 1. La problemática de las denominadas parejas mixtas, desde este punto de vista, debe ser estudiada más a fondo.

La otra característica sociodemográfica que nos interesaba analizar es el estado civil de agresores y victimas. Establecimos en nuestra hipótesis que las parejas de hecho presentarían más problemas de violencia en la pareja que las casadas, hecho que corroboran los datos. Básicamente la pregunta que se plantea en este caso es si la violencia de género está presente principalmente entre hombres y mujeres casados, si se produce entre las parejas de hecho o si se produce en situaciones de divorcio y/o separación de la pareja. Para ello hemos tenido en cuenta la variable lugar de nacimiento, estableciendo una triple distinción: aquellas parejas formadas por españoles, las parejas en las que agresor/a o víctima son migrantes -o parejas mixtas-y, por último, aquellas parejas en las que ambos son de origen extranjero (aunque el país de origen no sea el mismo). Podemos observar los resultados en la tabla número 2 . 
ANEXO 1

POBLACIÓN DE LA PROVINCIA DE ALMERÍA Y LA REGIÓN DE MURCIA SEGÚN LUGARES DE PROCEDENCIA (2010).

\begin{tabular}{|c|c|c|c|}
\hline & AMBOS SEXOS & VARONES & MUJERES \\
\hline \multicolumn{4}{|l|}{ TOTAL POBLACIÓN } \\
\hline Provincia de Almería & 695560 & 358112 & 337448 \\
\hline Región de Murcia & 1461979 & 738627 & 723352 \\
\hline \multicolumn{4}{|l|}{ NACIDOS EN ESPAÑA } \\
\hline Provincia de Almería & 541028 & 269112 & 271916 \\
\hline Región de Murcia & 1212431 & 599124 & 613307 \\
\hline \multicolumn{4}{|c|}{ NACIDOS EN EL EXTRANJERO } \\
\hline Provincia de Almería & 154532 & 89000 & 65532 \\
\hline Región de Murcia & 249548 & 139503 & 110045 \\
\hline \multicolumn{4}{|l|}{ PAISES EUROPEOS } \\
\hline Provincia de Almería & 75200 & 37699 & 37501 \\
\hline Región de Murcia & 81672 & 41597 & 40075 \\
\hline \multicolumn{4}{|l|}{ PAISES AFRICANOS } \\
\hline Provincia de Almería & 52612 & 38092 & 14520 \\
\hline Región de Murcia & 72852 & 50223 & 22629 \\
\hline \multicolumn{4}{|l|}{ PAISES AMERICANOS } \\
\hline Provincia de Almería & 24594 & 11879 & 12715 \\
\hline Región de Murcia & 90190 & 44743 & 45447 \\
\hline \multicolumn{4}{|l|}{ PAISES ASIATICOS } \\
\hline Provincia de Almería & 2085 & 1308 & 777 \\
\hline Región de Murcia & 4750 & 2886 & 1864 \\
\hline \multicolumn{4}{|l|}{ PAISES DE OCEANIA } \\
\hline Provincia de Almería & 41 & 22 & 19 \\
\hline Región de Murcia & 84 & 54 & 30 \\
\hline
\end{tabular}

Fuente: Padrón municipal 2010. Instituto Nacional de Estadística 
TABLA 2

TIPO DE RELACIÓN ENTRE AGRESOR/A Y VÍCTIMA EN LAS SENTENCIAS RELACIONADAS CON LA VIOLENCIA FAMILIAR EN LA PROVINCIA DE ALMERÍA Y LA REGIÓN DE MURCIA (ENERO 2005-JULIO 2010) (390 CASOS).

\begin{tabular}{|c|c|c|c|c|c|}
\hline $\begin{array}{l}\text { TIPO DE } \\
\text { RELACIÓN }\end{array}$ & $\begin{array}{l}\text { VÍCTIMA Y } \\
\text { AGRESOR/A } \\
\text { ESPAÑOLES }\end{array}$ & $\begin{array}{l}\text { VICTIMA O } \\
\text { AGRESOR/A } \\
\text { MIGRANTES }\end{array}$ & $\begin{array}{l}\text { VÍCTIMA Y } \\
\text { AGRESOR/A } \\
\text { MIGRANTES }\end{array}$ & $\begin{array}{l}\text { NO ESTAN } \\
\text { ASIGNADAS } \\
\text { AMBAS } \\
\text { NACIONALIDADES }\end{array}$ & TOTAL \\
\hline \multicolumn{6}{|l|}{ EN PAREJA } \\
\hline $\begin{array}{l}\text { Pareja de } \\
\text { hecho }\end{array}$ & $62(34,83 \%)$ & $16(51,6 \%)$ & $46(50,54 \%)$ & 36 & 160 \\
\hline Casados & $47(26,4 \%)$ & $5(16,12 \%)$ & $30(32,96 \%)$ & 22 & 104 \\
\hline $\begin{array}{l}\text { Novios sin } \\
\text { convivencia }\end{array}$ & $1(0,56 \%)$ & & $1(1,09 \%)$ & 2 & 4 \\
\hline \multicolumn{6}{|l|}{ EX PAREJA } \\
\hline $\begin{array}{l}\text { Ex pareja (no } \\
\text { casados) }\end{array}$ & $28(15,73 \%)$ & $6(19,35 \%)$ & $11(12,08 \%)$ & 17 & 62 \\
\hline Divorciados & $22(12,35 \%)$ & $3(9,67 \%)$ & $2(2,19 \%)$ & 5 & 32 \\
\hline $\begin{array}{l}\text { En trámites de } \\
\text { separación }\end{array}$ & $10(5,61 \%)$ & & & 3 & 13 \\
\hline $\begin{array}{l}\text { Separados } \\
\text { legalmente }\end{array}$ & $7(3,93 \%)$ & & $1(1,09 \%)$ & 3 & 11 \\
\hline $\begin{array}{l}\text { Separados de } \\
\text { hecho }\end{array}$ & $1(0,56 \%)$ & $1(3,22 \%)$ & & 2 & 4 \\
\hline Total & 178 & 31 & 91 & 90 & 390 \\
\hline
\end{tabular}

Fuente: Elaboración propia.

En lo referente a las relaciones entre víctima y agresor que aparecen en las sentencias, se observa que la relación predominante serían las parejas de hecho, seguidas de los matrimonios, tanto entre los españoles como entre los migrantes (Ver Tabla 2). Este dato corrobora el que se obtiene en la Macroencuesta (2011), donde se señala que las mujeres solteras que han sufrido violencia por parte de sus parejas en el último año suponen un 3,5\% del total de mujeres entrevistadas, mientras que las que estaban casadas suponían un $2,1 \%$. 


\subsection{Delitos por los cuales se condena en el ámbito de la violencia en la pareja o ex pareja}

El análisis de sentencias nos permite comparar también el tipo de delitos por los que son condenados españoles y migrantes. En principio, hemos supuesto que no habría una diferencia relevante entre ambas poblaciones. Nos fijaremos particularmente en el lugar de origen de los agresores varones y las victimas mujeres ${ }^{9}$. Hay que tener en cuenta que en muchos casos el agresor fue condenado por más de un delito. Además, hay que puntualizar que si comparamos el dato de delito por violencia en el ámbito familiar y lesiones en el ámbito familiar con los delitos de maltrato habitual se puede apreciar con claridad la tendencia de los tribunales a condenar los primeros. Esto es así porque el delito de violencia habitual supone hechos de mayor gravedad y, por tanto, una pena más gravosa para el condenado, implicando una mayor dificultad probatoria, ya que para apreciar la «habitualidad», los tribunales deben observar que el sujeto activo responda a situaciones de tensión familiar con maltrato como línea habitual de conducta. Además, los tribunales prácticamente no condenan por el delito de violencia psíquica habitual ${ }^{10}$.

En la tabla 3 se puede observar que el delito por el cual se condena en mayor número de ocasiones, tanto en los agresores españoles como migrantes, es el delito de malos tratos definido por el artículo 153 del código penal. En el 80\% de las sentencias donde el agresor y la víctima son migrantes se ha condenado por malos tratos ocasionales, frente al $65 \%$ de las sentencias en las que agresor y víctima son españoles. Es decir, a los migrantes se les condena, sobre todo, por malos tratos ocasionales, por lo que podemos decir que los datos refutan nuestra cuarta hipótesis.

Respecto al maltrato habitual, se puede observar cómo a los hombres migrantes se les condena menos que a los españoles por este delito. Es importante precisar que el maltrato habitual se presenta

9 Vamos a analizar las condenas de parejas formadas por agresores hombres y víctimas mujeres, puesto que constituyen el grupo del que tenemos más datos. Por este motivo, de los 390 casos hemos excluido las riñas en pareja y los casos en los que la agresora es mujer. Además no hemos podido incluir los casos en los que desconocíamos la nacionalidad de agresor o víctima (92 casos) y dos casos en los que se produce el sobreseimiento y archivo de las actuaciones.

10 Entre las resoluciones encontramos solamente un caso en el que los protagonistas son españoles. 
TABLA 3

DELITOS RELACIONADOS CON LA VIOLENCIA FAMILIAR EN LOS QUE LA VÍCTIMA ES MUJER EN LA PROVINCIA DE ALMERÍA Y LA REGIÓN DE MURCIA (ENERO 2005-JULIO 2010) (272 CASOS)

\begin{tabular}{|l|l|l|l|}
\hline & $\begin{array}{l}\text { VÍCTIMA Y AGRESOR/A } \\
\text { ESPAÑOLES }\end{array}$ & $\begin{array}{l}\text { VICTIMA O AGRESOR/A } \\
\text { MIGRANTES }\end{array}$ & $\begin{array}{l}\text { VICTIMA Y AGRESOR/A } \\
\text { MIGRANTES }\end{array}$ \\
\hline Amenazas/coacciones & $56(37,58 \%)$ & $10(45,45)$ & $11(14,10 \%)$ \\
\hline $\begin{array}{l}\text { Malos tratos ocasionales } \\
\text { y lesiones }\end{array}$ & $94(63,08 \%)$ & $19(86,36 \%)$ & $62(79,48 \%)$ \\
\hline $\begin{array}{l}\text { Maltrato físico o } \\
\text { psicológico habitual }\end{array}$ & $29(19,46 \%)$ & $4(18,18 \%)$ & $7(8,97 \%)$ \\
\hline $\begin{array}{l}\text { Agresiones sexuales/ } \\
\text { violaciones }\end{array}$ & $12(8,05 \%)$ & $2(9,09 \%)$ & $1(1,28 \%)$ \\
\hline Quebrantamientos & $14(9,39 \%)$ & $1(4,54 \%)$ & $1(1,28 \%)$ \\
\hline Otros (1) & $7(4,69 \%)$ & $2(9,09 \%)$ & 78 \\
\hline Total parejas & 149 & 22 & \\
\hline
\end{tabular}

Fuente: Elaboración propia.

Nota 1: En «otros» se incluye detención ilegal, abandono de familia e impago de pensiones, delito relativo a la prostitución denuncia falsa, resistencia a la autoridad, asesinato y tentativa de asesinato.

con frecuencia en las resoluciones combinado con otros delitos, como el delito de lesiones, las agresiones sexuales, las amenazas y las coacciones, entre otros. En general, en las parejas españolas se produce una mayor variedad de conductas delictivas -y de mayor intensidad- en comparación con las parejas migrantes y mixtas.

Como destacamos antes, el porcentaje de migrantes que aparecen en las sentencias es mucho mayor que el porcentaje de migrantes en la población residente en este territorio y que el porcentaje de mujeres extranjeras que sufren violencia en la pareja respecto al total de mujeres. Por tanto, el hecho de que haya muchos más malos tratos ocasionales entre los migrantes que entre los españoles corrobora la idea de que la violencia en la pareja entre poblaciones migrantes está siendo más observada ${ }^{11}$, que entre los españoles. Es

11 Es posible que en estas poblaciones se persigan más los actos delictivos. No obstante, también podría ocurrir que las mujeres migrantes tengan más problemas para declarar y probar el maltrato por falta de información, por la barrera lingüística y por la dificultad para hacer públicos asuntos «íntimos» como las agresiones sexuales. También puede incidir que en las poblaciones migrantes utilizan la asistencia jurídica gratuita. Sus abogados, en ocasiones, 
decir, el resultado de refutar la hipótesis cuarta es coherente con los resultados obtenidos para nuestra hipótesis general.

\subsection{Circunstancias específicas de los agresores/as: los antecedentes penales, consumo de alcohol, drogas y problemas psicológicos}

Respecto a los antecedentes penales, nuestra hipótesis afirmaba que los migrantes tendrían más probabilidades de reincidir que los españoles. Si observamos las sentencias ${ }^{12}$ podemos decir que, de entrada, es muy poco frecuente que migrantes y españoles tengan antecedentes penales.

\section{TABLA 4}

COMPARATIVA DE ANTECEDENTES PENALES EN LOS AGRESORES/AS MIGRANTES Y AUTÓCTONOS EN LAS SENTENCIAS RELACIONADAS CON LA VIOLENCIA FAMILIAR EN LA PROVINCIA DE ALMERÍA Y LA REGIÓN DE MURCIA (ENERO 2005-JULIO 2010) (328 DE 390 CASOS).

\begin{tabular}{|l|l|l|l|l|}
\hline & $\begin{array}{l}\text { HOMBRE } \\
\text { AGRESOR } \\
\text { ESPAÑOL }\end{array}$ & $\begin{array}{l}\text { HOMBRE } \\
\text { AGRESOR } \\
\text { MIGRANTE }\end{array}$ & $\begin{array}{l}\text { MUJER } \\
\text { AGRESORA } \\
\text { ESPAÑOLA }\end{array}$ & $\begin{array}{l}\text { MUJER } \\
\text { AGRESORA } \\
\text { MIGRANTE }\end{array}$ \\
\hline No tiene antecedentes & $127(64,79 \%)$ & $96(85,21 \%)$ & $12(92,3 \%)$ & $6(100 \%)$ \\
\hline Antecedentes cancelables y/o no computables & $29(14,79 \%)$ & $1(0,86 \%)$ & & \\
\hline Antecedentes por delito de malos tratos & $25(12,75 \%)$ & $10(8,69 \%)$ & $1(7,69)$ & \\
\hline Antecedentes por quebrantamiento de condena & $10(5,10 \%)$ & $1(0,86 \%)$ & & \\
\hline Antecedentes por amenazas & $10(5,10 \%)$ & $1(0,86 \%)$ & & \\
\hline Condenados por un delito anterior & $9(4,59 \%)$ & $4(3,47 \%)$ & & \\
\hline Medida cautelar de alejamiento & $5(2,55 \%)$ & $2(1,73 \%)$ & & \\
\hline Total & 196 & 113 & 13 & 6 \\
\hline
\end{tabular}

Fuente: Elaboración propia.

podrían prestar más atención a sus clientes particulares que a los del turno de oficio, disponiendo de menos tiempo para preparar sus defensas.

12 Debemos tener en cuenta que los agresores/as pueden incluirse en más de una categoría, puesto que, por ejemplo, pueden no tener antecedentes pero sí medidas cautelares o pueden tener antecedentes por varios delitos, etc. 
No obstante, como se observa en la tabla 4, se producen diferencias entre los autóctonos y los migrantes respecto a la reincidencia, siendo menos frecuente en el colectivo de migrantes (un 85,21\% de los agresores migrantes no tiene antecedentes penales) que entre los españoles (un 64,9\%), por lo que se refutaría nuestra hipótesis quinta, pues los migrantes extranjeros, de hecho, reinciden menos que los españoles.

Nuestros resultados podrían explicarse teniendo en cuenta que los migrantes están sobredimensionados en las resoluciones judiciales, por lo que de nuevo daríamos crédito a los resultados obtenidos para nuestra hipótesis general. Pero además, habría que tener en cuenta que los agresores migrantes han podido tener que volver a sus países tras el maltrato, además de por la sustitución de la pena privativa de libertad por la expulsión del territorio nacional, por el requisito de carecer de antecedentes penales a la hora de renovar los permisos de residencia que, tras la sentencia de condena, les haría entrar en una situación de irregularidad administrativa. Pueden también haber huido a sus países para evitar la condena.

TABLA 5

PROBLEMAS PSICOLÓGICOS, CONSUMO DE ALCOHOL Y DROGAS EN LOS AGRESORES/AS MIGRANTES Y AUTÓCTONOS EN LAS SENTENCIAS RELACIONADAS CON LA VIOLENCIA FAMILIAR EN LA PROVINCIA DE ALMERÍA Y LA REGIÓN DE MURCIA (ENERO 2005-JULIO 2010) (51 DE 328 CASOS)

\begin{tabular}{|l|l|l|l|}
\hline & $\begin{array}{l}\text { HOMBRE AGRESOR } \\
\text { ESPAÑOL }\end{array}$ & $\begin{array}{l}\text { HOMBRE AGRESOR } \\
\text { MIGRANTE }\end{array}$ & $\begin{array}{l}\text { MUJER AGRESORA } \\
\text { ESPAÑOLA }\end{array}$ \\
\hline Alcohol & $22(11,22 \%)$ & $11(9,73 \%)$ & $1(7,69 \%)$ \\
\hline Drogas & $17(8,67 \%)$ & $1(0,88 \%)$ & \\
\hline Problemas psicológicos & $7(3,57 \%)$ & & $1(7,69 \%)$ \\
\hline Total agresores & 196 & 113 & 13 \\
\hline
\end{tabular}

Fuente: Elaboración propia

Nos interesaba obtener información sobre los problemas de alcohol, drogas y psicológicos que detectamos en los casos de violencia en el ámbito familiar ${ }^{13}$. Las toxicomanías se han considerado

13 Encontramos estas circunstancias en 59 de los 390 casos analizados. 
como un factor que se presenta con frecuencia en los casos de violencia en la pareja. Al analizar esta circunstancia, tal y como se observa en la tabla $n^{\circ} 5$, podemos afirmar que la presencia de toxicomanías en los agresores en nuestro análisis es minoritaria, aunque significativa, tanto entre los migrantes como entre los españoles.

\subsection{Análisis de los lugares dónde sucede y los testigos que presencian la violencia}

Aunque no tenemos referencias de estudios donde se analizan los lugares en los que se produce la violencia en la pareja entre migrantes y autóctonos, las resoluciones judiciales sí nos informan sobre los lugares donde ocurre la violencia al hacer referencia a los hechos probados durante el proceso judicial. Hemos supuesto que, tanto en el caso de las poblaciones migrantes como entre la española, la violencia en la pareja tendría lugar en el hogar y en presencia de los hijos/as como principales testigos, circunstancias que corroboran los datos, aunque podemos llevar a cabo algunas especificaciones.

Evidentemente, los espacios de la violencia domestica reflejan los lugares donde españoles y migrantes conviven con sus parejas habitualmente. En ambas poblaciones, el lugar que se señala como más frecuente en los episodios de violencia en la pareja es el domicilio común. Pero el porcentaje es un poco más alto en el caso de las migrantes ${ }^{14}$. Los/as españolas hablan de una segunda vivienda, y comentan que la violencia sucedió en su casa de campo, apartamento en la playa, etc.

14 Respecto a este apartado debemos de concretar que el número de casos en que se concretaba el lugar donde suceden las agresiones es de 300. Sin embargo no hemos podido analizar 61 casos porque las nacionalidades de agresor y víctima no han sido concretadas. En algunos casos, se encuentra más de un lugar donde la violencia fue ejercida. 
TABLA 6

LUGAR DONDE SE PRACTICÓ LA VIOLENCIA FAMILIAR EN LAS SENTENCIAS RELACIONADAS CON LA VIOLENCIA FAMILIAR EN EL ÁMBITO TERRITORIAL DE ALMERÍA Y MURCIA (ENERO 2005-JULIO 2010) (239 CASOS DE 300)

\begin{tabular}{|l|l|l|l|}
\hline & $\begin{array}{l}\text { VÍCTIMA Y AGRESOR/A } \\
\text { ESPAÑOLES }\end{array}$ & $\begin{array}{l}\text { VICTIMA O AGRESOR/A } \\
\text { MIGRANTES }\end{array}$ & $\begin{array}{l}\text { VICTIMA Y AGRESOR/A } \\
\text { MIGRANTES }\end{array}$ \\
\hline Domicilio familiar & $69(38,76 \%)$ & $19(61,29 \%)$ & $50(54,94 \%)$ \\
\hline Domicilio de la víctima & $20(11,23 \%)$ & $2(6,45 \%)$ & $2(2,19 \%)$ \\
\hline $\begin{array}{l}\text { Domicilio de familiares o } \\
\text { amigos (1) }\end{array}$ & $5(2,8 \%)$ & $3(9,67 \%)$ & $2(2,19 \%)$ \\
\hline Lugar de trabajo & $5(2,8 \%)$ & $1(3,22 \%)$ & $2(2,19 \%)$ \\
\hline vehículo privado (2) & $3(1,68 \%)$ & $3(9,67 \%)$ & $3(3,29 \%)$ \\
\hline calle (3) & $20(11,23 \%)$ & $4(12,9 \%)$ & $10(10,98 \%)$ \\
\hline establecimiento público (4) $)$ & $14(7,86 \%)$ & & $5(5,49 \%)$ \\
\hline llamada telefónica & $2(1,12 \%)$ & & $1(1,09 \%)$ \\
\hline Total casos & 138 & 32 & 75 \\
\hline $\begin{array}{l}\text { Total parejas en las que } \\
\text { se especifica el lugar }\end{array}$ & 137 & 30 & 72 \\
\hline
\end{tabular}

Fuente: Elaboración propia.

Nota 1: Entre los familiares se incluye el padre o madre y los hermanos/as.

Nota 2: Entre los vehículos se incluyen turismos, furgonetas y camiones.

Nota 3: En este apartado nos referimos a avenidas y calles, plazas, parques, paseo marítimo y portal del domicilio.

Nota 4. Como establecimientos públicos encontramos un colegio, hotel, bingo, centro comercial, restaurante, bar, locutorio, discoteca, piscina, mercadillo, pabellón deportivo, banco y centro de mayores. También hemos incluido el autobús por ser un medio de transporte público.

Además, encontramos bastantes referencias a establecimientos públicos. Al respecto, queremos matizar que el único establecimiento público señalado tanto por autóctonos como por migrantes es el autobús. En el resto de establecimientos encontramos diferencias entre los migrantes, que señalaron lugares como un hotel, locutorio, piscina y mercadillo, y los autóctonos que apuntaron al resto de lugares especificados: bingo, centro comercial, restaurante, discoteca, pabellón deportivo, banco y centro de mayores.

Por último, hay que señalar que las resoluciones judiciales nos informan también sobre los testigos que presencian la violencia ${ }^{15}$.

15 Se encuentran 172 casos en los que la violencia es presenciada por algún testigo. Sin embargo, hemos tenido que prescindir de 41 casos porque 
Como se puede deducir al observar la tabla 7, en la violencia en el ámbito familiar adquiere importancia la familia, que suele ser testigo directo, y cuyos miembros presencian la violencia en mayor proporción que el resto de testigos. En un segundo plano encontraríamos otras personas que formarían parte de la red social de las víctimas o agresores, que son testigos en un menor número de ocasiones y pueden ser testigos directos o de referencia. Los testigos que no mantienen relación alguna con agresor o víctima suelen serlo de referencia y se presentan en un número menor de casos.

TABLA 7

TESTIGOS QUE PRESENCIAN LA VIOLENCIA FAMILIAR EN LAS SENTENCIAS RELACIONADAS CON LA VIOLENCIA FAMILIAR EN EL ÁMBITO TERRITORIAL DE ALMERÍA Y MURCIA (ENERO 2005-JULIO 2010) (131 CASOS DE 300).

\begin{tabular}{|l|l|l|l|}
\hline & $\begin{array}{l}\text { VÍCTIMA Y AGRESOR/A } \\
\text { ESPAÑOLES }\end{array}$ & $\begin{array}{l}\text { VICTIMA O AGRESOR/A } \\
\text { MIGRANTES }\end{array}$ & $\begin{array}{l}\text { VICTIMA } \text { Y AGRESOR/A } \\
\text { MIGRANTES }\end{array}$ \\
\hline Madre/padre & $11(6,17 \%)$ & & $5(5,49 \%)$ \\
\hline Hijos/as & $41(23,03 \%)$ & $4(12,9 \%)$ & $12(13,18 \%)$ \\
\hline Hermanos/as & $6(3,37 \%)$ & & $6(6,59 \%)$ \\
\hline Otros familiares (1) & $3(1,68 \%)$ & $1(3,22 \%)$ & \\
\hline Pareja actual & $1(0,56 \%)$ & & \\
\hline Familia política & $2(1,12 \%)$ & $1(3,22 \%)$ & $5(5,49 \%)$ \\
\hline Amigos/as & $8(4,49 \%)$ & $1(3,22 \%)$ & $2(2,19 \%)$ \\
\hline Vecinos/as & $8(4,49 \%)$ & & $4(4,39 \%)$ \\
\hline Guardia Civil, Policía & $9(5,05 \%)$ & $3(9,67 \%)$ & $9(9,89 \%)$ \\
\hline Compañero/a de trabajo & $3(1,68 \%)$ & & $1(1,09 \%)$ \\
\hline Compañero/a de piso & & & $3(3,29 \%)$ \\
\hline Otros & $3(1,68 \%)$ & $1(3,22 \%)$ & $1(1,09 \%)$ \\
\hline Total casos & 82 & 9 & 40 \\
\hline Total parejas & 178 & 31 & 91 \\
\hline
\end{tabular}

Fuente: Elaboración propia.

Nota 1: sobrinos/as y abuelos.

Nota 2: cuñado/a, suegra, hermano del cuñado/a.

Nota 3: vigilante, cliente de un bar, conductor de autobús y portero.

desconocemos la nacionalidad de ambos miembros de la pareja. En algunas ocasiones la violencia es presenciada por más de una persona. Los porcentajes se han calculado sobre el total de parejas de las cuales conocemos la nacionalidad. 
Los hijos de la pareja son los que más frecuentemente observan el episodio violento, tanto en el caso de la población española como de la migrante. Pero esa situación se produce con mucha más frecuencia en el colectivo de españoles-as. Entre la población migrante se encuentra más la circunstancia de que sean los hermanos o familia política quienes presencien la violencia. Entre los testigos que mantienen otro tipo de relación con el agresor o la víctima, encontramos los/as vecinos/as, amigos/as, compañeros/as de trabajo y de piso. Sobresale la poca cantidad de testigos que hay al margen de los familiares y el hecho de que los compañeros de piso sean testigos solo entre migrantes. Por último encontramos testigos que no mantienen ningún tipo de relación con el agresor o víctima, entre los cuales encontramos a la Policía local o nacional y Guardia Civil. Son más frecuentes este tipo de testigos entre los migrantes que entre los españoles.

\section{CONCLUSIONES Y DISCUSIÓN}

En nuestra investigación hemos tratado de explorar la manera en que las resoluciones judiciales reflejan la violencia en la pareja y expareja entre las poblaciones autóctona y migrante. Partíamos de la hipótesis de que las resoluciones judiciales que se producen en un territorio sobre violencia en la pareja no tienen por qué corresponderse con la frecuencia o incluso las características de ese fenómeno social (Calvo, 1995:9; Guemureman y Jorolinsky, 2007:17). Y, efectivamente, hemos demostrado que las resoluciones judiciales dictadas en las provincias de Murcia y Almería (2005-10) reflejan que la violencia en la pareja es sufrida con mayor frecuencia por las poblaciones migrantes que por la española. Pero, además, amplifican el fenómeno, pues tienden a sobre-representarlo. Es decir, hay muchas más resoluciones judiciales en el caso de las poblaciones migrantes de lo que se esperaría si atendemos a los datos de la incidencia real de dicha violencia.

La segunda conclusión es que las resoluciones judiciales sobre violencia en la pareja detectan violencia de mujeres hacia varones. En este sentido, se podría decir que avisan sobre la existencia de violencia que, aunque minoritaria, habría que investigar, tanto entre las poblaciones migrantes como entre la española. En este caso, habría que discutir si tiene $-\mathrm{O}$ no- sentido desarrollar 
Macroencuestas sobre violencia en la pareja que entrevisten exclusivamente a mujeres.

En tercer lugar, hemos observado que las resoluciones judiciales no se dictan en la misma proporción respecto a todos los grupos de migrantes. En el caso de Almería y Murcia, la población procedente de América resulta ser la más sobre-representada, lo que podría corroborar otros estudios que han hallado especificidades en la incidencia de la violencia en la pareja entre las poblaciones de minorías étnicas (Dutton et al. 2000; Kugel et al. 2009; Moracco, et al., 2005 en latinos; Gondolf, 2005 en afroamericanos). En el caso de Almería, este análisis coincidiría con los resultados de un estudio cualitativo que destaca el alto nivel de conflictividad que se produce en las parejas procedentes de América Latina, en comparación con las que proceden de África y Europa del Este (Herranz et al. 2010). Las resoluciones judiciales, además, avisan de la existencia de parejas mixtas, pues la cantidad de hombres y mujeres - agresores y víctimas- no se corresponde con la cantidad de hombres y mujeres de una misma procedencia.

En tercer lugar, el estado civil de agresores y víctimas que aparece en las resoluciones judiciales se corresponde con los estudios disponibles (Holliday y Lucas, 2010), al señalar que la violencia en la pareja, tanto en las poblaciones migrantes como entre la española, es más frecuente entre las parejas de hecho que entre las casadas. La mayor vulnerabilidad derivada de la falta de documentación, la inserción en un mercado laboral secundario, la falta de red social, etc. hacen que las parejas de hecho de migrantes se encuentren con más conflictos de género en los que los hombres hacen uso de la violencia contra sus compañeras. Entre los migrantes, además, las situaciones de violencia apenas se producen entre quienes están divorciados, separados legalmente o en proceso de separación.

En cuarto lugar hemos concluido que a los migrantes se les condena en mayor proporción que a los españoles por malos tratos ocasionales que por maltrato habitual. Eso, unido al hecho de que los españoles suelen ser condenados por un abanico de delitos más amplio, y el hecho de que los migrantes apenas son condenados por amenazas o delitos sexuales como violación, hace pensar que la violencia en la pareja se percibiría más por los tribunales entre la población migrante que entre la española.

Pero es que, además, del análisis de las sentencias se desprende que los agresores migrantes tienen en menos ocasiones antecedentes 
penales que los españoles. Se puede argumentar que las características de los migrantes (más jóvenes, recién llegados, el hecho de que puedan ser expulsados del país por lo que se reduciría la posibilidad de reincidencia) puedan explicar esa diferencia. Pero también se puede argumentar que este resultado esté simplemente corroborando el hecho de que las poblaciones migrantes -y particularmente los agresores migrantes- estén sobre-representados en las sentencias en comparación con los porcentajes de incidencia de malos tratos, además de que son juzgados principalmente por malos tratos ocasionales.

Respecto al consumo de drogas y problemas psicológicos, hemos observado que aparece como un fenómeno minoritario entre los agresores que aparecen en las resoluciones judiciales, tanto en el caso de los españoles como de los migrantes, por lo que nuestro estudio podría refutar los resultados obtenidos por Gondolf (2005).

La violencia en la pareja que aparece reflejada en las resoluciones judiciales tiene lugar en el domicilio común, tanto entre la población migrante como entre la española, y los hijos/as son los principales testigos. Las resoluciones judiciales avisan de que puede que los lugares donde ocurre la violencia en la pareja varíen según el lugar de procedencia de los protagonistas. Los testigos que la observan, aparte de los hijos, nos llevarían a la misma conclusión.

\section{LIMITACIONES DEL ESTUDIO}

Nuestro estudio, aunque innovador, presenta múltiples limitaciones. Por un lado está el hecho de que no disponemos de buenos datos sobre la incidencia real de la violencia en la pareja que nos permitan llevar a cabo análisis en profundidad sobre cuánta, cómo, dónde y en presencia de quién, tiene lugar dicha violencia en las poblaciones migrante y española. Eso hace que no podamos establecer con más precisión hasta qué punto las resoluciones judiciales reflejan, sobredimensionan o infra dimensionan lo que ocurre en la realidad social. Con los resultados que hemos obtenido podemos señalar que las sentencias se pronuncian más sobre casos de violencia en la pareja cuyos protagonistas son migrantes.

Por otro lado, habría que considerar quién es el responsable de que haya proporcionalmente más resoluciones judiciales respecto a la población migrante que a la española. Si bien es cierto que 
algunos jueces hacen más valoraciones morales que otros, consideramos necesario poner de manifiesto que sería un error afirmar que hay una amplia discrecionalidad judicial en las resoluciones dictadas en nuestro país, puesto que, al margen de que los magistrados incluyan en los fundamentos de derecho más o menos retórica, el fallo de su sentencia debe tener en cuenta múltiples elementos legales que limitan su margen de decisión que, en todo caso, debe basarse en la interpretación de la norma - y todavía más en el derecho penal, que es lógicamente más restrictivo en su aplicación debido a las consecuencias que tiene-. Es más, una sentencia que no basa su fallo en estos términos sería fácilmente recurrible. Desde nuestro punto de vista, habría que estudiar más elementos relacionados con el sistema judicial — cantidad de denuncias, órdenes de protección dictadas, etc- para poder determinar por qué se escriben proporcionalmente más sentencias sobre violencia en la pareja relacionadas con la población migrante que con la española.

Por último, nos gustaría destacar el hecho de que todavía no contamos con una base de datos donde se puedan consultar todas las resoluciones judiciales respecto a la violencia en la pareja. Pero, además, en las resoluciones judiciales no aparece reflejado el país de origen de las personas a las que se refiere la sentencia. Eso dificulta enormemente el trabajo de los y las investigadoras que nos vemos obligadas a reconstruir, uno por uno, los lugares de origen de víctimas y agresores/as.

\section{BIBLIOGRAFÍA}

Amnistía Internacional (2005): Inmigrantes indocumentadas, ¿Hasta cuándo sin protección frente a la violencia de género? Madrid, (Recuperado de la Web: www.es.amnesty.org el día 20 de agosto de 2010).

- (2008): Obstinada realidad, derechos pendientes. Tres años de la Ley de Medidas de Protección Integral contra la violencia de género. (Extraído de la Web: http://www.es.amnesty.org/index.php?id=3054 el día 3/11/2010).

- (2012): ¿Qué justicia especializada? A siete años de la Ley Integral contra la Violencia de Género: obstáculos al acceso y obtención de justicia y protección.

BARRÉre, M. A. (2008): «Género, discriminación y violencia contra las mujeres». En Laurenzo, P. Madueda, M. L. y Rubio, A. Género, violencia y derecho. Valencia, Tirant lo Blanch alternativa, pp. 27-48. 
Calvo, M. (1995): «La investigación socio-juridica en España: estado actual y perspectivas». En Bergalli, R. ¿Para qué la Sociología jurídica en España?, Inst. Internacional de Sociología Jurídica, p. 17-46.

- (2001): El tratamiento de la violencia doméstica en la Administración de Justicia. Ed. Universidad de Zaragoza.

Cear-EusKadi (2009): Persecución por motivos de género y derecho de asilo: del contexto global al compromiso local. El sistema de asilo español frente a la violación de los derechos humanos de las mujeres y de lesbianas, gays, bisexuales y transexuales. Bilbao.

Cederborg, A. C. y Lamb, M. E. (2006): «How does the legal system respond when children with learning difficulties are victimized?»Child Abuse \& Neglect, 30, pp. 537-547.

CIS - Secretaría de Estado de Servicios Sociales e Igualdad (2012): Macroencuesta de violencia de género 2011.

Cole, B. A. (2009): "Gender, narratives and intersectionality: can personal experience approaches to research contribute to "undoing gender"?»International Review of Education, 55, pp. 561-578.

Colectivo IOÉ (1991): Trabajadoras extranjeras de servicio doméstico en Madrid. Ginebra, Oficina Internacional del Trabajo.

Consejo de Europa (1985): Recomendación $(85,4)$, adoptada por el Comité de Ministros el 26 de marzo de 1985, sobre la violencia dentro de la Familia.

Dekeseredy, W. y Schwartz, M. D. (2002): «Theorizing Public Housing Woman Abuse as a Function of Economic Exclusion and Male Peer Support». Women's Health and Urban Life: An International and Interdisciplinary Journal, 1(2), pp. 26-45.

Dutton, M., Orloff, L. Hass, G. (2000): «Characteristics of help-seeking behaviors, resources, and service needs of battered immigrant Latinas: Legal and policy implications», Georgetown Journal on Poverty Law and Policy, 7, pp. 245-305.

Expósito, F., Herrera, A. y Herrera, M. C. (2008): «Ley integral contra la violencia de género: Análisis de sentencias». En RODRÍGUEZ, F.J. et. Al. Psicología jurídica: entorno judicial y delincuencia. Ediciones de la Universidad de Oviedo.

García, F. J. (2009): «La investigación social sobre violencia de género: una propedéutica». En Miranda, M. J., Martín, M. T. y Marugán, B. Amor, Razón, Violencia. Madrid, Los libros de la Catarata, pp. 55-84.

Gondolf, E. (2005): «Culturally-Focused Battered Counselling for AfricanAmerican Men». TxostenaViolence and Victimization Research Division National Institute of Justice, www.iup.edu/maati/publications.

Guemureman, S. y Jorolinsky, K. (2007): «Sentencias judiciales ¿Qué hay detrás de esa decisión?» Trabajo presentado en el XXVII Congreso ALAS (México, 2007), Grupo de Trabajo nº 22 Sociología de la Infancia y Juventud / Socialización y violencia. 
Halliday, J. y Lucas, A. (2010): «Economic Factors and Relationship Quality Among Young Couples: Comparing Cohabitation and Marriage». Journal of Marriage and Family, 72.

Heise, L. L. y García-Moreno, C. (2003): "La violencia en la pareja», en Krug, LL. Dahlberg, K. A. Mercy, A. B. Zwi y LOzano, R. Informe mundial sobreviolencia y salud (97-131 orr), Organización Panamericana de la Salud, Washingon DC.

Herranz, G., Fernandez, J. S., Rodriguez, P., Checa, J. C., Fernández, I., GonZalez, M. J., Ainz, A. y Muñoz, J. M. (2010): Sexo-género e inmigración: Consensos y conflictos en Almería. Granada, Alhulia.

Holgado, I. (2008): «Trabajos y estrategias económicas de las mujeres migrantes» en Rodríguez, P. Mujeres, trabajos y empleos en tiempos de globalización. Barcelona, Icaria, pp. 87-115.

Johnson M. P. (1995): «Patriarchal terrorism and common couple violence: Two forms of violence against women». Journal of Marriage and the Family, 57, 2, pp. 283-294.

Kingsnorth, R. F. y Macintosh, R. C. (2007): «Intimate Partner Violence: The Role of Suspect Gender in Prosecutorial Decision-Making». Justice Quarterly, 24, 3, pp. 460-495.

Kugel, C. Et al. (2009): «Familias con Voz: Community Survey Results from an Intimate Partner Violence (IPV) Prevention Project with Migrant Workers». J Fam Viol, 24, pp. 649-660.

LARRAuri, E. (2007): Criminología crítica y violencia de género. Madrid, Trota.

LOMBARDo, E. (2009): «La institucionalización de la "interseccionalidad” del género con otras desigualdades en la unión europea: desarrollos políticos y contestaciones». IX Congreso español de ciencia política y de la administración. Málaga, 23-25 septiembre de 2009.

Malley-Morrison, K. y Hines, D. (2007): «Attending to the Role of Race/Ethnicity in Family Violence Research», Journal of Interpersonal Violence, 22, pp. 943-972.

Manzanos, C. (2004): «Factores sociales y decisiones judiciales». Sociológica, 5, pp. 127-159.

MenjÍvar, C. y Salcido, O. (2002): «Women and Domestic Violence: Common Experiences in Different Countries». Gender and Society, 16, 6, pp. 898-920.

Minai, M. (2010): «Judicial review and transitional justice: reflective judgment in three contexts». Papeles del CEIC, 60, pp. 1-29.

Mohanty, C. T. (2008): «Bajo los Ojos de Occidente: Feminismo Académico y Discursos Coloniales». En Suárez, L. y Hernández, R. A. (coord.) Descolonizando el Feminismo: Teorías y Prácticas desde los Márgenes. Madrid, Cátedra, pp. 112-162.

Moracco, K., Hilton, A., Hodges, K. y Eta Frasier, P. (2005): «Knowledge and Attitudes About Intimate Partner Violence Among Immigrant Latinos in Rural North Carolina: Baseline Information and Implications for Outreach», Violence Against Women, 11, pp. 337-352. 
Morokvasic, M. (2007): «Migración, Género, Empoderamiento». En Lenz, I. Ullrich, C. y Fersch, B. Gender Orders Unbound. Globalisation, Restructuring and Reciprocity. Opladen, Farmington Hills.

ONU (1993): Resolución 48/104 de la Asamblea General de la ONU, Declaration on the elimination of violence against Women, 20 de diciembre de 1993.

Oso, L. (1998): La migración hacia España de mujeres jefas de hogar. Madrid, Instituto de la Mujer, Ministerio de Trabajo y Asuntos Sociales.

PARElla, S. (2003): Mujer, inmigrante y trabajadora: la triple discriminación. Barcelona, Anthropos.

RaJ, A. y Silverman, J. (2002): «Violence against Immigrant Women: The Roles of Culture, Context, and Legal Immigrant Status on Intimate Partner Violence». Violence Against Women, 8, pp. 367-398.

Rubio, C. (2004): «La violencia machista en contextos multiculturales», Seminario de Violencia contra las mujeres e Inmigración. Gasteiz.

Secretario General de Naciones Unidas (2006): Estudio a fondo sobre todas las formas de violencia contra la mujer.

SheA, A. H. y Bagshaw, D. (2008): «The idealised post-separation family in Australian family law: A dangerous paradigm in cases of domestic violence». Journal of Family Studies, 14, pp. 291-309.

Sigma Dos (2006): III Macroencuesta sobre la violencia contra las mujeres. Madrid, Secretaría General de Políticas de Igualdad. Instituto de la Mujer.

Sokoloff, N. J. y Dupont, I. (2005): «Domestic Violence at the Intersections of Race, Class, and Gender». Violence against Women, 11, 1, pp. 38-64.

VARMA, K. N. (2002): «Exploring “youth” in court: An analysis of decisionmaking in youth court bail hearings». Canadian Journal of Criminology; 44, 2, pp. 143-164.

West, C. (2005): «Domestic violence in ethnically and racially diverse families: The "political gag order" has been lifted», en Richie, B. E., SokoloFFETA, N. J., Pratt, C. Domestic violence at the margins: Readings on race, class, gender, and culture. NJ: Rutgers University Press, New Brunswick. 
